\title{
A NEW ROBUST KALMAN FILTER ALGORITHM UNDER OUTLIERS AND SYSTEM UNCERTAINTIES
}

\author{
S. C. Chan, Z. G. Zhang and K. W. Tse \\ Department of Electrical and Electronic Engineering, The University of Hong Kong \\ Pokfulam Road, Hong Kong.
}

\begin{abstract}
This paper proposes a new robust Kalman filter algorithm under outliers and system uncertainties. The robust Kalman filter of Durovic and Kovacevic is extended to include unknown-but-bounded parameter uncertainties in the state or observation matrix. We first formulate the robust state estimation problem as an M-estimation problem, which leads to an unconstrained nonlinear optimization problem. This is then linearized and solved iteratively as a series of linear least-squares problem. These least-squares problems, subject to the bounded system uncertainties using the robust least squares method proposed by A. Ben-Tal and A. Nemirovski. Simulation results show that the new algorithm leads to a better performance than the conventional algorithms under outliers as well as system uncertainties.
\end{abstract}

\section{INTRODUCTION}

Since its introduction in the 60's, the Kalman filter has found numerous applications in automatic control, navigation, and communications. The original Kalman filter addresses the optimal state estimation of a linear state space system in the least mean squares sense with state and measurement uncertainties modeled by Gaussian random processes. Since the mean squares performance criterion is closely to the assumption that the state density is Gaussian distributed, its performance will degrade significantly when the state or measurement noises are non-Gaussian. A frequently encountered class of distribution of interest in practice is impulsive noise or outliers with a much heavier tails than the Gaussian distribution. They occur either naturally or in other man-made sources. Although it is possible to generalize the Kalman filter to more general distributions other than the Gaussian distribution, the estimation of their parameters is usually quite complicated, especially for transient disturbances with impulsive nature. In order to make the Kalman filtering algorithm more robust against such outliers, several algorithms based on the concept of robust statistics have been proposed. Masreliez and Martin [3] first applied the min-max score function approach in robust statistics originally proposed by Huber [4] to combat the outliers. However, the linear transformation proposed in [3] does not generally exist and is somewhat difficult to apply. Recently, Durovic and Kovacevic [1] established the equivalence between the Kalman filter algorithm and a particular least-squares regression problem. In other words, the conventional Kalman filter problem can also be viewed and solved as this linear least-squares problem. Further, the use of the leastsquares criterion is equivalent to saying that the noise involved in the least-squares problem is Gaussian distributed. This further suggests that if the state or measure noises exhibit impulsive behavior, a better solution is to replace the least-squares estimation by more robust M-estimation technique in robust statistics. Durovic and Kovacevic also employed the method introduced by Myers and Tapley [5] method to estimate the unknown a priori state and observation noise statistics in the robust Mestimation framework. Besides the robust statistics approach, which is targeted to the detection and suppression of transient outliers, another focus in robust Kalman filter research is to address the uncertainties of the state-space models arising from imprecise measurements and nonlinear effects. Since these effects are sometimes very difficult to model precisely, it is desirable to have the Kalman filter robust to bounded variations or uncertainties in the system parameters which are simpler to quantify in practice. In the control community, the stability problem is of paramount importance, and thus the Kalman filter should optimize not only the given performance criteria, but also the stability margin under the given and bounded system uncertainties. This gives rise to a number of robust Kalman filter algorithms [6, 7, and more] and most of them are associated with the solving of a set of linear matrix inequalities (LMI) or a semi-definite programming (SDP) problem.

To the best knowledge of the authors, robust Kalman filters that address both the outliers and system uncertainties have not been studied before. In this paper, we shall focus on the robust state estimation problem with outliers and system uncertainties using the Kalman filter framework. More precisely, we shall extend the robust Kalman filter of Durovic and Kovacevic to include unknown-but-bounded parameter uncertainties in observation matrix of the system model. Similar to [1], we first formulate the robust state estimation problem as an M-estimation problem, which leads to an unconstrained nonlinear optimization problem. This is then linearized and solved iteratively as a series of linear least-squares problem. We then solve these least-squares problems, subject to the bounded system uncertainties using the robust least squares method proposed by A. Ben-Tal and A. Nemirovski [2]. Since the robust least-squares problem can be formulated as a SDP problem, it gives rise to an iterative algorithm, involving a SDP at each step, which usually terminates in a few steps. The paper is organized as follows. Section 2 briefly reviews the formulation of the conventional Kalman filter as a certain linear regression problem. Section 3 is devoted to the robust Kalman filter based on M-estimation for outlier suppression. Section 4 extends this algorithm to include system uncertainties, based on the SDP formulation of the robust least squares problem. The effectiveness of the proposed algorithm is demonstrated by simulation results and comparison in Section 5. Conclusions are drawn in Section 6. 


\section{KALMAN FILTER AND LINEAR REGRESSION}

The discrete Kalman filter is a set of mathematical equations to estimate the state $\underline{x}(k)$ of a linear discrete-time state-space model:

$$
\begin{aligned}
& \underline{x}(k+1)=\mathbf{F}(k) \underline{x}(k)+\mathbf{G}(k) \underline{w}(k) \\
& \underline{z}(k)=\mathbf{H}(k) \underline{x}(k)+\underline{v}(k)
\end{aligned}
$$

where $\underline{x}(k), \underline{z}(k), \underline{w}(k)$, and $\underline{v}(k)$ are respectively the state vector, observation vector, state noise, and observation noise at time instant $k . \mathbf{F}(k), \mathbf{G}(k)$, and $\mathbf{H}(k)$ are the state transition matrix, state noise transition matrix and observation matrix, respectively. $\underline{w}(k)$ and $\underline{v}(k)$ are assumed to be uncorrelated white noises with covariances:

$$
E\left\{\left[\begin{array}{c}
\underline{w}(j) \\
\underline{v}(j)
\end{array}\right]\left[\begin{array}{ll}
\underline{w}^{T}(k) & \underline{v}^{T}(k)
\end{array}\right]^{T}\right\}=\operatorname{diag}\left(\mathbf{Q}(k) \delta_{j k}, \mathbf{R}(k) \delta_{j k}\right),
$$

where $E\{\cdot\}$ is the expectation and $\delta_{j k}$ is the Kronecker's delta symbol ( $\delta_{j k}=0$ for $j \neq k$ and $\delta_{j k}=1$ for $j=k$ ).

If the state density is Gaussian distributed, then the optimal state estimator in the least mean squares criterion can be computed by the Kalman filter. Let $\hat{x}(\mathrm{k} / \mathrm{m})$ ( $m=k-1, k)$ represent the estimator of $\underline{x}(k)$ given the measurement up to time instant $m\{z(j), j \leq m\}$, and $P(k / m)$ the corresponding error covariance matrix of $\underline{x}(k)$. The standard Kalman filter recursions are:

$$
\begin{aligned}
& \underline{\hat{x}}(k+1 / k)=\mathbf{F}(k) \underline{\hat{x}}(k+1 / k) \\
& \mathbf{P}(k+1 / k)=\mathbf{F}(k) \mathbf{P}(k / k) \mathbf{F}^{T}(k)+\mathbf{G}(k) \mathbf{Q}(k) \mathbf{G}^{T}(k) \\
& \underline{e}(k)=\underline{z}(k)-\mathbf{H}(k) \underline{\hat{x}}(k / k-1) \\
& \mathbf{K}(k)=\mathbf{P}(k / k-1) \mathbf{H}^{T}(k)\left[\mathbf{H}(k) \mathbf{P}(k / k-1) \mathbf{H}^{T}(k)\right. \\
& \quad+\mathbf{R}(k)]^{-1} \\
& \underline{\hat{x}}(k / k)=\underline{\hat{x}}(k / k-1)+\mathbf{K}(k) \underline{e}(k) \\
& \mathbf{P}(k / k)=[\mathbf{I}-\mathbf{K}(k) \mathbf{H}(k)] \mathbf{P}(k / k-1),
\end{aligned}
$$

where $\underline{e}(k)$ is the prediction error of the observation vector, and its covariance matrix is $\mathbf{H}(k) \mathbf{P}(k / k-1) \mathbf{H}^{T}(k)+\mathbf{R}(k)$.

Combining (1) and (2) together, we get the following equivalent linear model:

$$
\left[\begin{array}{c}
\mathbf{I} \\
\mathbf{H}(k)
\end{array}\right] \underline{x}(k)=\left[\begin{array}{c}
\mathbf{F}(k-1) \underline{x}(k-1 / k-1) \\
\underline{z}(k)
\end{array}\right]+\mathbf{e}(k) .
$$

where I is the identity matrix,

$$
\begin{aligned}
& \mathbf{e}(k)=\left[\begin{array}{c}
\mathbf{F}(k-1)[\underline{x}(k-1)-\underline{\hat{x}}(k-1 / k-1)]+\mathbf{G}(k-1) \underline{w}(k-1) \\
-\underline{v}(k)
\end{array}\right] \\
& \text { and } \quad E\left[\mathbf{e}(k) \mathbf{e}^{T}(k)\right]=\left[\begin{array}{cc}
\mathbf{P}(k / k-1) & \mathbf{0} \\
\mathbf{0} & \mathbf{R}(k)
\end{array}\right]=\mathbf{S}(k) \mathbf{S}^{T}(k)
\end{aligned}
$$

$\mathbf{S}(k)$ can be obtained by computing the UD factorization or Cholesky decomposition of $E\left[\boldsymbol{e}(k) \boldsymbol{e}^{T}(k)\right]$ where $\mathbf{P}(k / k-1)$ can be obtained from (5). Multiplying both sides of (10) by $\mathbf{S}^{-1}(k)$, we get

$$
\mathbf{Y}(k)=\mathbf{X}(k) \underline{\beta}(k)+\xi(k),
$$

where $\quad \mathbf{X}(k)=\mathbf{S}^{-1}(k)\left[\begin{array}{c}\mathbf{I} \\ \mathbf{H}(k)\end{array}\right], \quad \xi(k)=-\mathbf{S}^{-1}(k) \mathbf{e}(k)$, $\underline{\beta}(k)=\underline{x}(k)$, and $\mathbf{Y}(k)=\mathbf{S}^{-1}(k)\left[\begin{array}{c}\hat{x}(k / k-1) \\ \underline{z}(k)\end{array}\right]$.

Note that, after the multiplying $\mathbf{S}^{-1}(k)$ operation, the residual $\xi(k)$ are now whiten, since $E\left[\xi(k) \xi^{T}(k)\right]=\mathbf{I}$. (11) is nothing but a standard least-squares regression with solution given by [1]:

$$
\underline{\hat{\beta}}(k)=\left(\mathbf{X}^{T}(k) \mathbf{X}(k)\right)^{-1} \mathbf{X}^{T}(k) \mathbf{Y}(k),
$$

and covariance

$E\left[(\underline{\beta}(k)-\underline{\hat{\beta}}(k))(\underline{\beta}(k)-\underline{\hat{\beta}}(k))^{T}\right]=\left(\mathbf{X}^{T}(k) \mathbf{X}(k)\right)^{-1}$.

In other words, the Kalman filter can also be thought of as the solution to a weighted least-squares problem with $\underline{\hat{\beta}}(k)=\underline{\hat{x}}(k / k)$ and $P(k / k)=\operatorname{cov}(\underline{\hat{\beta}}(k))$. Both the Kalman filter and the linear least-squares regression are based on the Gaussian assumption, so their performance will deteriorate considerably when the observation or state noise are contaminated by non-Gaussian impulsive noise. In the next section, we will introduce the robust form of (12) to improve the robustness of the state estimation.

\section{IMPROVED ROBUST KALMAN FILTER AGAINST OUTLIERS}

Equation (12) is solved by minimizing the following least-squares cost function $J_{n}(\underline{\beta})$ :

$$
\begin{aligned}
\underline{\hat{\beta}}(k) & =\arg \min _{\underline{\beta}} J_{n}(\underline{\beta})=\arg \min _{\underline{\beta}} \sum_{i=1}^{n}\left|e_{i}(k)\right|^{2} \\
& =\arg \min _{\underline{\beta}} \sum_{i=1}^{n}\left(y_{i}(k)-x_{i}{ }^{T}(k) \underline{\beta}\right)^{2}
\end{aligned}
$$

where $y_{i}(k)$ is the $i$-th element of $\mathbf{Y}(k), x_{i}{ }^{T}(k)$ is the $i$-th row of $\mathbf{X}(k), e_{i}(k)$ is the corresponding estimation error, and $n$ is the dimension of $\mathbf{Y}(k)$.

In M-estimation, a nonlinear score function $\rho(\cdot)$ instead of the quadratic function in (14) is minimized to restrain the adverse effect of outliers:

$$
\begin{aligned}
\underline{\hat{\beta}}(k) & =\arg \min _{\underline{\beta}} J_{n}(\underline{\beta})=\arg \min _{\underline{\beta}} \sum_{i=1}^{n} \rho\left(e_{i}(k)\right) \\
& =\arg \min _{\underline{\beta}} \sum_{i=1}^{n} \rho\left(y_{i}(k)-x_{i}^{T}(k) \underline{\beta}\right)
\end{aligned}
$$

A commonly used function for $\rho(\cdot)$ is the Huber function:

$$
\rho(e)=\left\{\begin{array}{ll}
\xi|e|-\xi^{2} / 2, & |e| \geq \xi \\
e^{2} / 2, & |e|<\xi
\end{array},\right.
$$

where $\xi$ is the threshold parameter which should be carefully selected so that the estimation error $e_{i}$ can be deemphasized when $\left|e_{i}\right|>\xi$ as shown in Fig. 1. Equating the first partial derivatives with respect to the elements of $\underline{\hat{\beta}}(k)$, say $\hat{\beta}_{j}(k), j=1, \cdots, p$, to zero, we obtain the following necessary condition for optimality: 


$$
\sum_{i=1}^{n} x_{i j}(k) \Psi\left(y_{i}(k)-x_{i}{ }^{T}(k) \underline{\hat{\beta}}(k)\right)=0, j=1, \cdots, p
$$

where $x_{i j}(k)$ is the element in the $i$-th row and $j$-th column of $\mathbf{X}(n)$ and $\Psi(\cdot)=\rho^{\prime}(\cdot)$ as shown in Fig.1. Durovic and Kovacevic gave a weighted least-squares solution to solve (17) and here we use the iterative reweighted least-squares (IRLS) method to achieve more precise solution.

1. Choose a starting value for $\underline{\hat{\beta}}_{0}(k): \underline{\hat{\beta}}_{0}(k)=\underline{\hat{\beta}}(k-1)$.

2. Compute the estimation residuals:

$$
e_{s, i}(k)=y_{i}(k)-x_{i}^{T}(k) \underline{\hat{\beta}}_{s, i}(k) .
$$

3. Calculate the updated estimate of $\underline{\hat{\beta}}_{s+1}(k)$ by

$$
\underline{\hat{\beta}}_{s+1}(k)=\left(\mathbf{X}^{T}(k) \boldsymbol{\Omega}_{s}(k-1) \mathbf{X}(k)\right)^{-1} \mathbf{X}^{T}(k) \boldsymbol{\Omega}_{s}(k-1) \mathbf{Y}(k),
$$$$
\text { where } \boldsymbol{\Omega}_{s}(k-1)=\operatorname{diag}\left\{\omega_{s, 1}(k-1), \cdots, \omega_{s, n}(k-1)\right\} \text {, }
$$

$$
\omega_{s, i}(k-1)=\Psi\left(e_{s, i}(k)\right) / e_{s, i}(k) \text {. }
$$

4. Use the updated $\underline{\hat{\beta}}_{s+1}(k)$ to step 2 to get the new residuals $e_{s+1}(k)$ and repeat the process until converge.

Experiments showed a good and fast converge of the proposed algorithm. Generally, the number of iterations was about $s=3 \sim 5$ and never exceeded 10 . The threshold parameters $\xi$ in (16) can be determined by the method proposed in [8]. $\xi_{i}$ can be chosen as $\xi_{i}=2.576 \hat{\sigma}_{e_{i}}(k)$, where $\hat{\sigma}_{e_{i}}(k)$ is the estimated standard deviation of the estimation error. The robust estimation of $\hat{\sigma}_{e_{i}}(k)$ can be implemented by

$$
\hat{\sigma}_{e_{i}}^{2}(k)=\lambda_{e} \hat{\sigma}_{e_{i}}^{2}(k-1)+c_{1}\left(1-\lambda_{e}\right) \operatorname{med}\left(A_{e_{i}}(k)\right),
$$

where $c_{1}=1.483\left(1+5 /\left(N_{w}-1\right)\right) \quad$ is a finite sample correction factor, med $(\cdot)$ is the median operator, $A_{e_{i}}(k)=\left\{e_{i}^{2}(k), \ldots, e_{i}^{2}\left(k-N_{w}+1\right)\right\}, \lambda_{e}$ is the forgetting factor and $N_{w}$ is the length of the estimation time window.

\section{ROBUST KALMAN FILTER AGAINST SYSTEM UNCERTAINTY}

As mentioned earlier, the matrices $\mathbf{F}, \mathbf{G}$, and $\mathbf{H}$ might be subject to uncertainties, which are more convenient in practice to be modeled as unknown-but bounded structured parametric uncertainty. A "robust" Kalman filter should then minimize the upper hound of the least-squares estimation error in the presence of these uncertainties. The least-squares formulation of the Kalman filter in (11)-(13) allows us to solve this problem as a robust least squares problem with structured parametric uncertainty, which has received considerable attention recently in SDP community.

Assume that the observation matrix can be represented as $\mathbf{H}=\mathbf{H}_{\mathbf{0}}+\Delta \mathbf{H}$, where $\mathbf{H}_{0}$ is the known observation matrix and $\Delta \mathbf{H}$ is the unknown but bounded uncertainty, which is assumed to be given by the following structured model:

$$
\begin{aligned}
\Delta \mathbf{H} & =\sum_{i=1}^{l} u_{H_{i}} \mathbf{H}_{i} \\
U_{H} & =\left\{(\mathbf{H}) \mid \exists u_{H} \in \mathbf{R}^{l}, u_{H}{ }^{T} u_{H} \leq 1: \mathbf{H}=\mathbf{H}_{0}+\sum_{i=1}^{l} u_{H_{i}} \mathbf{H}_{i}\right\} .
\end{aligned}
$$

$\boldsymbol{H}_{i}$ are the vertices of the polytope, which contains the uncertainty $\Delta \mathbf{H}$. From (11) and $\mathbf{X}(k)=\mathbf{S}^{-1}(k)\left[\begin{array}{c}\mathbf{I} \\ \mathbf{H}(k)\end{array}\right]$, we can see that there is a corresponding uncertainty in $\mathbf{X}$ and $\mathbf{Y}$ :

$$
\mathbf{Y}(k)=(\mathbf{X}(k)+\Delta \mathbf{X}(k)) \underline{\beta}(k)+\xi(k)+\Delta \xi(k) .
$$

and they can be modeled in a similar form as follows:

$$
\begin{aligned}
& U=\left\{(\mathbf{X}, \mathbf{Y}) \mid \exists u \in \mathbf{R}^{l}, u^{T} u \leq 1:\right. \\
& \left.\quad[\mathbf{X} ; \mathbf{Y}]=\left[\mathbf{X}_{0}, \mathbf{Y}_{0}\right]+\sum_{i=1}^{l} u_{i}\left[\mathbf{X}_{i} ; \mathbf{Y}_{i}\right]\right\}, \\
& \Delta \mathbf{X}=\sum_{i=1}^{l} u_{i} \mathbf{X}_{i}, \text { and } \Delta \mathbf{Y}=\sum_{i=1}^{l} u_{i} \mathbf{Y}_{i} .
\end{aligned}
$$

Because of the uncertainties in $\mathbf{X}(k)$ and $\mathbf{Y}(k)$, any particular choice $\underline{\beta}$ would lead to many different residual norms: $\|(\mathbf{X}+\Delta \mathbf{X}) \underline{\hat{\beta}}-(\mathbf{Y}+\Delta \mathbf{Y})\|_{2}$, one for each possible choice of $\mathbf{X}$ and $\mathbf{Y}$ in the area $\mathbf{X}+\Delta \mathbf{X}$ and $\mathbf{Y}+\Delta \mathbf{Y}$, respectively. We want to choose an estimate $\underline{\hat{\beta}}$ that minimizes the maximum possible residual norm. That is: minimize maximize $\quad\|(\mathbf{X}+\Delta \mathbf{X}) \underline{\hat{\beta}}-(\mathbf{Y}+\Delta \mathbf{Y})\|_{2}$, or equivalently:

minimize $\delta$,

subject to $\quad\|(\mathbf{X}+\Delta \mathbf{X}) \underline{\hat{\beta}}-(\mathbf{Y}+\Delta \mathbf{Y})\|_{2} \leq \delta$.

It can be shown in [2] this so-called robust least squares problem with the uncertainty model in (23) can be expressed in a set linear matrix inequalities (LMIs) or semidefinite programming (SDP) problem. More precisely, the robust feasible solution of $\underline{\beta}$ can be solved if and only if there exist real $\delta$ and $\mu$ that the triplet $(\beta, \delta, \mu)$ satisfies the following LMIs:

$\left(\begin{array}{c|c|ccc}\delta \mathbf{I} & \mathbf{X}_{0} \underline{\beta}-\mathbf{Y}_{0} & \mathbf{X}_{1} \underline{\beta}-\mathbf{Y}_{1} & \cdots & \mathbf{X}_{l} \underline{\beta}-\mathbf{Y}_{l} \\ \hline\left[\mathbf{X}_{0} \underline{\beta}-\mathbf{Y}_{0}\right]^{T} & \delta-\mu & & \\ \hline\left[\mathbf{X}_{1} \underline{\beta}-\mathbf{Y}_{1}\right]^{T} & & \mu & \\ \cdots & & \ddots & \\ {\left[\mathbf{X}_{l} \underline{\beta}-\mathbf{Y}_{l}\right]^{T}} & & & & \mu\end{array}\right) \geq 0 \cdot$

The geometric interpretation of the least-squares formulation with the given uncertainty model is illustrated in Fig. 2. The two pentagons represent the two possible areas of $\mathbf{X}(k)$ and $\mathbf{Y}(k)$, respectively. The optimal estimation of $\beta$ is not the least-squares solution obtained "from point to point", but "from area to area". 


\section{SIMULATION RESULTS}

Our simulation system is a three-state track model with the following parameters:

$$
\mathbf{F}=\left[\begin{array}{ccc}
1.01 & 0 & 0 \\
0 & 1.01 & 0 \\
0 & 0 & 1.01
\end{array}\right], \quad \mathbf{G}=\left[\begin{array}{l}
0.1 \\
0.2 \\
0.5
\end{array}\right], \quad \mathbf{H}=\left[\begin{array}{lll}
1+\delta & 0 & -1
\end{array}\right] .
$$

The first element in $\mathbf{H}$ has a parametric uncertainty $|\delta|<0.5$ and $\mathbf{H}_{0}=\left[\begin{array}{lll}1 & 0 & -1\end{array}\right]$. The state noise $w(k)$ is assumed to be zero-mean white Gaussian noise with a variance of 0.001 , and the random observation noise $v(k)$ is generated from the following contaminated Gaussian noise model with probability distribution function (p.d.f.):

$$
\begin{aligned}
& p(\cdot) \sim(1-\varepsilon) \cdot N(\cdot \mid 0,0.01)+\varepsilon \cdot N\left(\cdot \mid 0, \sigma_{0}{ }^{2}\right)^{3}, \\
& 0 \leq \varepsilon \leq 1, \sigma_{0}{ }^{2}>1 .
\end{aligned}
$$

Here, $\varepsilon=0$ corresponds to Gaussian noise and a value of $\varepsilon=0.15$ and $\sigma_{0}=10$ is used to simulate the non-Gaussian noise and outliers. Simulation results are compared in terms of the cumulative estimation error (CEE):

$$
C E E(k)=\frac{1}{k} \sum_{i=1}^{k} \frac{\|\underline{\hat{\beta}}(i)-\underline{\beta}(i)\|_{2}}{\|\underline{\beta}(i)\|_{2}} .
$$

Fig. 3 shows the results of the various algorithms in Gaussian noise. We can see that the difference between the conventional Kalman filter and the robust Kalman filter is very small. This indicates the robust Kalman filter also works well in Gaussian noise environment. However, it can be seen from Fig. 4 that in non-Gaussian noise environment, the robust Kalman filter against outliers can achieve a much better performance. We also see that, in both noise circumstances, the robust Kalman filter with system uncertainty gives the best performance because prior information of the system uncertainty has been taken into account in the robust least squares framework.

\section{CONCLUSION}

A new robust Kalman filter algorithm under outliers and system uncertainties is presented. It extends the robust Kalman filter of Durovic and Kovacevic to include unknown-but-bounded parameter uncertainties in the state or observation matrix. The robust state estimation problem is first formulated as a M-estimation problem, which leads to a unconstrained nonlinear optimization problem. This is solved iteratively as a series of linear least-squares problem subject to the bounded system uncertainties using the robust least squares method proposed by A. Ben-Tal and A. Nemirovski. The improved robustness of the proposed algorithm is illustrated by computer simulation results.

\section{REFERENCE}

[1] Z. M. Durovic and B. D. Kovacevic, "Robust Estimation with Unknown Noise Statistics," IEEE Transactions on Automatic Control, vol. 44, Issue 6, pp. 1292-1296, June 1999.

[2] A. Ben-Tal and A. Nemirovski, Lectures on Modern Convex Optimization. Philadelphia, PA: SIAM, 2001.

[3] C. J. Masreliez and R. D. Martin, "Robust Bayesian estimation for linear model and robustifying the Kalman filter," IEEE Trans. Automat. Contr., vol. 22, pp. 361-371, 1977.

[4] P. J. Huber, Robust Statistics. New York: Wiley, 1973.
[5] K. A. Myers and B. D. Tapley, "Adaptive sequential estimation with unknown noise statistics," IEEE Trans. Automat. Contr., vol. 21, pp. 520-523, 1976.

[6] L. Li, Z. Q. Luo, T. N. Davidson, K. M. Wong, and E. Bossé, "Robust filtering via semi-definite programming with applications to target tracking", SIAM J. Optim., vol. 12, pp. 740755, Dec., 2002.

[7] F. Wang and V. Balakrishnan, "Robust Kalman Filters for Linear Time-Varying Systems with Stochastic Parametric Uncertainties." IEEE Trans. Signal Processing, vol. 50, no. 4, pp. 803-813, April 2002.

[8] Y. Zou, S. C. Chan and T. S. Ng, "Robust M-estimate adaptive filtering," IEE Proc.-Vis. Image Sig. Proc., vol. 148, No. 4, August2001.
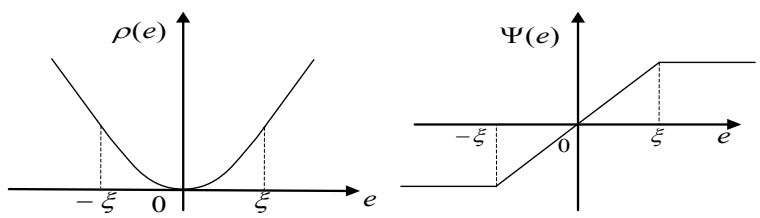

Fig. 1: Huber function $\rho(e)$ and $\Psi(e)$.

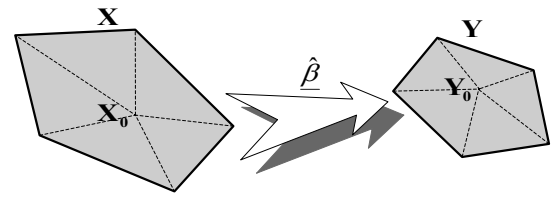

Fig. 2: Geometric interpretation of the robust least-squares formulation with uncertainty.

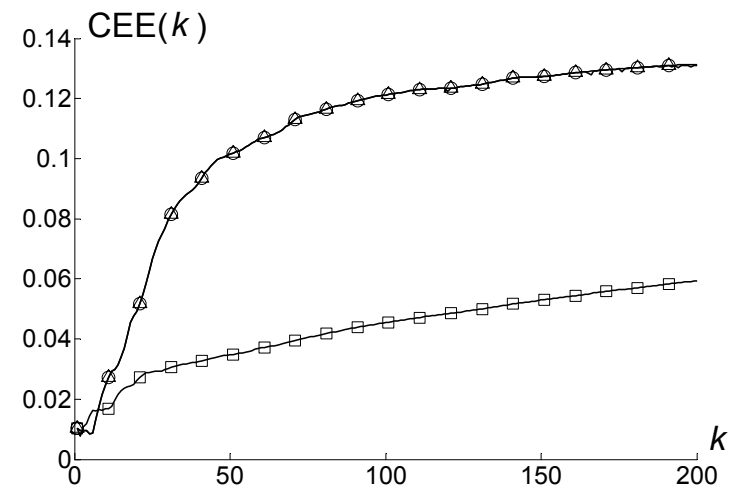

Fig. 3: Estimation error comparison in Gaussian noise: conventional Kalman filter (line with triangles), robust Kalman filter against outliers (line with circles), and robust Kalman filter against uncertainty (line with squares).

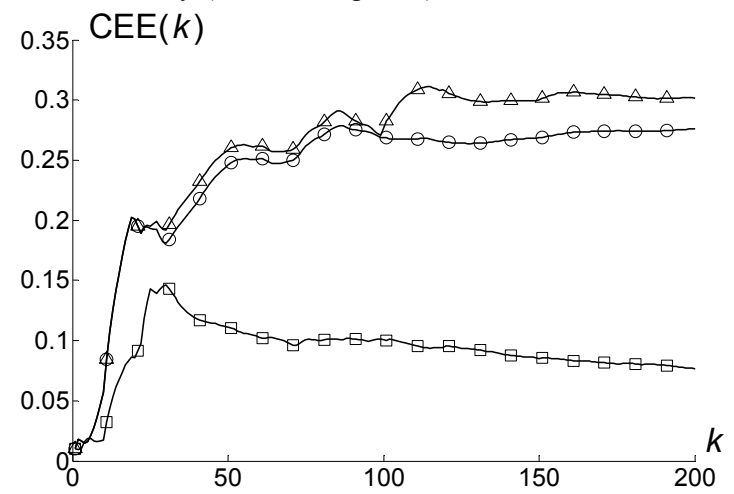

Fig. 4: Estimation error comparison in non-Gaussian noise: conventional Kalman filter (line with triangles), robust Kalman filter against outliers (line with circles), and robust Kalman filter against uncertainty (line with squares). 